\title{
NONLINEAR SCHRÖDINGER EQUATION WITH A WHITE-NOISE POTENTIAL: PHASE-SPACE APPROACH TO SPREAD AND SINGULARITY
}

\author{
ALBERT C. FANNJIANG
}

\begin{abstract}
We propose a phase-space formulation for the nonlinear Schrödinger equation with a white-noise potential in order to shed light on two issues: the rate of spread and the singularity formation in the average sense. Our main tools are the energy law and the variance identity. The method is completely elementary.

For the problem of wave spread, we show that the ensemble-averaged dispersion in the critical or defocusing case follows the cubic-in-time law while in the supercritical and subcritical focusing cases the cubic law becomes an upper and lower bounds respectively.

We have also found that in the critical and supercritical focusing cases the presence of a whitenoise random potential results in different conditions for singularity-with-positive-probability from the homogeneous case but does not prevent singularity formation. We show that in the supercritical focusing case the ensemble-averaged self-interaction energy and the momentum variance can exceed any fixed level in a finite time with positive probability.
\end{abstract}

\section{INTRODUCTION}

High-intensity laser beams propagating in dielectric media such as optical fibers, films or air are important problems both from fundamental and practical perspectives. The physical process is nonlinear and the amplitude modulation $\Psi$ is customarily described by a nonlinear Schrödinger equation with an additional inhomogeneous potential $V$ representing the random impurities in the medium.

Let $z$ and $\mathbf{x} \in \mathbb{R}^{d}$ be the longitudinal and transverse coordinates of the wave beam. In the physical setting, the transverse dimension $d$ may be $0,1,2$. The simplest non-dimensional form of the nonlinear Schrödinger (NLS) equation with a random potential $V$ then reads [1]

$$
i \frac{\partial}{\partial z} \Psi+\frac{\gamma}{2} \Delta_{\mathbf{x}} \Psi+\gamma^{-1} g|\Psi|^{2 \sigma} \Psi+\gamma^{-1} V \circ \Psi=0, \quad \sigma>0 .
$$

Similar equations also arise in many other contexts such as in plasma physics and the Bose-Einstein condensation, see [13] and references therein. Here we have written the equation in the comoving reference frame at the group velocity and non-dimensionalized the equation with the longitudinal and transverse reference lengths $L_{z}, L_{x} ; \gamma=L_{z} /\left(k L_{x}^{2}\right)$ is the Fresnel number; $g$ is nonlinear coupling coefficient with $g>0$ representing the self-focusing case and $g<0$ the self-defocusing case. For a nonlinear Kerr medium $\sigma=1$ leading to the cubic NLS equation. We shall let $\sigma$ be an arbitrary positive constant. We shall consider white-noise-in- $z$, $\mathbf{x}$-homogeneous random potential $V$ with

$$
\left\langle V(z, \mathbf{x}) V\left(z^{\prime}, \mathbf{x}^{\prime}\right)\right\rangle=\delta\left(z-z^{\prime}\right) \int e^{i\left(\mathbf{x}-\mathbf{x}^{\prime}\right) \cdot \mathbf{p}} \Phi(\mathbf{p}) d \mathbf{p}
$$

Research partially supported by the Centennial Fellowship from the American Mathematical Society, the UC Davis Chancellor's Fellowship and U.S. National Science Foundation grant DMS 0306659. 
where $\Phi(\mathbf{p})$ is the power spectral density and the product $V \circ \Psi$ in eq. (1) stands for the Stratonovich product. Here and below the bracket $\langle\cdot\rangle$ denotes the ensemble average w.r.t. the random medium. In view of the real-valuedness of $V$ we may assume

$$
\Phi(-\mathbf{p})=\Phi(\mathbf{p}), \quad \forall \mathbf{p} \in \mathbb{R}^{d}
$$

Such a short-range-in- $z$ potential arises in long-distance propagation when the longitudinal length $L_{z}$ of the wave beam is much larger than the correlation length of the random impurities in the medium resulting in rapidly fluctuating potential in the non-dimensionalized coordinates. And it is well known that in the short-range-correlation scaling limit a non-white-in- $z$ multiplicative noise gives rise to a Stratonovich, instead of Itô, integral with respect to a white-in- $z$ noise [12.

We are particularly interested in the problems of wave spread and singularity formation. To this end we shall use the phase-space approach of the Wigner distribution which is particularly useful in the regime of low Fresnel number $\gamma \ll 1$ which can be viewed as a high-frequency limit. The main ingredient of our analysis is the phase-space variance identity by which we derive various estimates for the mean spread, defined in (9), including precise behavior in the defocusing $g \leq 0$ or critical case $d \sigma=2$. To our knowledge, these are significant improvement over previous results (e.g. 11, 14, 3]) which are mostly for the linear problem. Wave spread is an important physical quantity in itself and in technological applications such as estimating the effect of nonlinearity on the information capacity of optical fiber communications [8, 10.

Using the variance identity we further show that the scattering by the random potentials results in conditions for singularity-with-positive-probability that are different from those for the homogeneous case.

In the present setting the physical roles of $t$ and $z$ are interchanged: the variable $t$ is space-like while the variable $z$ is time-like and we will refer to it as "time" occasionally, especially in the discussion of finite-time singularity.

\section{WigneR DistribUtion}

We consider the Wigner distribution of the form

$$
W(z, \mathbf{x}, \mathbf{p})=\frac{1}{(2 \pi)^{d}} \int e^{-i \mathbf{p} \cdot \mathbf{y}} \Psi\left(z, \mathbf{x}+\frac{\gamma \mathbf{y}}{2}\right) \Psi^{*}\left(z, \mathbf{x}-\frac{\gamma \mathbf{y}}{2}\right) d \mathbf{y}
$$

which is always real-valued and may be thought of as quasi-probability density on the phase space [9] [6. First its marginals are essentially the position and momentum densities

$$
\begin{aligned}
\int W(z, \mathbf{x}, \mathbf{p}) d \mathbf{p} & =|\Psi|^{2}(z, \mathbf{x}) \equiv \rho(z, \mathbf{x}) \\
\frac{1}{(2 \pi)^{d}} \int W(z, \mathbf{x}, \mathbf{p}) d \mathbf{x} & =\frac{1}{\gamma^{d}}|\hat{\Psi}(z, \mathbf{p})|^{2}
\end{aligned}
$$

which are nonnegative; indeed any marginal on a $d$-dimensional subspace is nonnegative everywhere. Also, the energy flux is given by

$$
\frac{1}{2 i}\left(\Psi \nabla \Psi^{*}-\Psi^{*} \nabla \Psi\right)=\int_{\mathbb{R}^{d}} \mathbf{p} W(z, \mathbf{x}, \mathbf{p}) d \mathbf{p} .
$$

In the whole phase space, however, the Wigner distribution is not everywhere non-negative in general and hence can not be a true probability density. As $\gamma \rightarrow 0$, the Wigner distribution has a nonnegative-measure-valued weak limit, called the Wigner measure [9]. Therefore the Wigner distribution is particularly useful for analyzing high frequency behaviors of waves. 


\section{The EVOlution EQUations}

From the NLS eq. (11) it is straightforward to derive the closed-form (Wigner-Moyal) equation for the Wigner distribution in the Itô sense [4, [5]

$$
d_{z} W+\mathbf{p} \cdot \nabla_{\mathbf{x}} W d z+\mathcal{U}_{\gamma} W d z-\mathcal{Q}_{\gamma} W d z+\mathcal{V}_{\gamma} W d z=0
$$

Here the self-adjoint operator $\mathcal{Q}_{\gamma}$ is the Stratonovich correction term

$$
\begin{aligned}
& \mathcal{Q}_{\gamma} W(z, \mathbf{x}, \mathbf{p}) \\
& \quad=\int \Phi(\mathbf{q}) \gamma^{-2}[-2 W(z, \mathbf{x}, \mathbf{p})+W(z, \mathbf{x}, \mathbf{p}-\gamma \mathbf{q})+W(z, \mathbf{x}, \mathbf{p}+\gamma \mathbf{q})] d \mathbf{q}
\end{aligned}
$$

and $\mathcal{U}_{\gamma}$ and $\mathcal{V}_{\gamma}$ are the nonlinear and linear Moyal operators, respectively

$$
\begin{aligned}
& \mathcal{U}_{\gamma} W(z, \mathbf{x}, \mathbf{p}) \\
& \quad=i \int e^{i \mathbf{q} \cdot \mathbf{x}} \gamma^{-1}[W(z, \mathbf{x}, \mathbf{p}+\gamma \mathbf{q} / 2)-W(z, \mathbf{x}, \mathbf{p}-\gamma \mathbf{q} / 2)] \widehat{U}(z, \mathbf{q}) d \mathbf{q}, \\
& \mathcal{V}_{\gamma} W(z, \mathbf{x}, \mathbf{p}) \\
& \quad=i \int e^{i \mathbf{q} \cdot \mathbf{x}} \gamma^{-1}[W(z, \mathbf{x}, \mathbf{p}+\gamma \mathbf{q} / 2)-W(z, \mathbf{x}, \mathbf{p}-\gamma \mathbf{q} / 2)] \widehat{V}(d z, d \mathbf{q})
\end{aligned}
$$

with

$$
U=g \rho^{\sigma} .
$$

Here we have somewhat abused the notation by writing $\hat{U}$ as the Fourier-transform-in-x of the function $U(z, \cdot)$ and $\hat{V}(d z, d \mathbf{q})$ as the spectral measure of the white-noise potential $V(z, \cdot)$. The spectral measure $\hat{V}(d z, d \mathbf{p})$ is related to the power spectral density $\Phi$ of $V$ as

$$
\left\langle\hat{V}(d z, d \mathbf{p}) \hat{V}^{*}\left(d z^{\prime}, d \mathbf{q}\right)\right\rangle=\delta\left(z-z^{\prime}\right) \delta(\mathbf{p}-\mathbf{q}) \Phi(\mathbf{p}) d z d \mathbf{p} d \mathbf{q}
$$

An important property of these integral operators is

$$
\int \mathcal{Q}_{\gamma} W d \mathbf{p}=\int \mathcal{U}_{\gamma} W d \mathbf{p}=\int \mathcal{V}_{\gamma} W d \mathbf{p}=0
$$

which will be useful in deriving the energy law and the variance identity.

A major advantage of formulating the wave process on the phase space using the Wigner distribution is that the (high-frequency) low-Fresnel number limit $\gamma$ can be easily obtained. Formally we see that as $\gamma \rightarrow 0$

$$
\begin{aligned}
& \mathcal{U}_{\gamma} W(z, \mathbf{x}, \mathbf{p}) \rightarrow \mathcal{U}_{0} W(z, \mathbf{x}, \mathbf{p}) \equiv \nabla_{\mathbf{x}} U(z, \mathbf{x}) \cdot \nabla_{\mathbf{p}} W(z, \mathbf{x}, \mathbf{p}) \\
& \mathcal{V}_{\gamma} W(z, \mathbf{x}, \mathbf{p}) \rightarrow \mathcal{V}_{0} W(z, \mathbf{x}, \mathbf{p}) \equiv \nabla_{\mathbf{x}} W(z, \mathbf{x}) \cdot \nabla_{\mathbf{p}} W(z, \mathbf{x}, \mathbf{p}) \\
& \mathcal{Q}_{\gamma} W(z, \mathbf{x}, \mathbf{p}) \rightarrow \mathcal{Q}_{0} W(z, \mathbf{x}, \mathbf{p})=\nabla_{\mathbf{p}} \cdot \mathbf{D} \nabla_{\mathbf{p}} W(z, \mathbf{x}, \mathbf{p})
\end{aligned}
$$

with the diffusion coefficient

$$
\mathbf{D}=\int \Phi(\mathbf{p}) \mathbf{p} \otimes \mathbf{p} d \mathbf{p}
$$

We shall refer to eq. (15) as the nonlinear Wigner-Moyal -Itô (NWMI) equation when $\gamma>0$ and as the nonlinear Liouville-Itô (NLI) equation when $\gamma=0$.

Another advantage of working with eq. (5) is that one can use it to evolve the mixed-state initial condition. The mixed-state Wigner distribution is a convex combination of the pure-state Wigner distributions (3) described as follows. 
Let $\left\{\Psi_{\alpha}\right\}$ be a family of $L^{2}$ functions parametrized by $\alpha$ which is weighted by a probability measure $P(d \alpha)$. Denote the pure-state Wigner distribution (3) by $W\left[\Psi_{\alpha}\right]$. A mixed-state Wigner distribution is given by

$$
\int W\left[\Psi_{\alpha}\right] P(d \alpha)
$$

The limiting set, as $\gamma \rightarrow 0$, of the mixed state Wigner distributions constitute the nonnegative Wigner measures [6], 9]. The NWMI or NLI equation preserves the mixed-state form (8) .

3.1. Basic properties. The NWMI and NLI equations conserve the total mass, i.e.

$$
\frac{\partial}{\partial z} N=0, \quad N=\int W(z, \mathbf{x}, \mathbf{p}) d \mathbf{x} d \mathbf{p}
$$

and the $L^{2}$ norm

$$
\frac{\partial}{\partial z} \int W^{2}(z, \mathbf{x}, \mathbf{p}) d \mathbf{x} d \mathbf{p}=0
$$

One can absorb the effect of the total mass $N$ into $g$ by the obvious rescaling of $W$ in the eq. (5). Henceforth we assume that $N=1$.

Let $S_{x}$ and $S_{p}$ be the spreads (or variances) of position and momentum, respectively

$$
\begin{aligned}
S_{x}(z) & =\int|\mathbf{x}|^{2} W(z, \mathbf{x}, \mathbf{p}) d \mathbf{x} d \mathbf{p} \\
S_{p}(z) & =\int|\mathbf{p}|^{2} W(z, \mathbf{x}, \mathbf{p}) d \mathbf{x} d \mathbf{p} .
\end{aligned}
$$

A natural space of initial data and solutions is the subspace $\mathcal{W} \subset L^{2}\left(\mathbb{R}^{2 d}\right)$ with a finite Dirichlet form

$$
-\int W \mathcal{Q}_{\gamma} W d \mathbf{x} d \mathbf{p}<\infty
$$

and finite, positive (pre-ensemble-averaged) variances $S_{x} \in(0, \infty), S_{p} \in(0, \infty)$. In addition, we shall also assume that the initial data have a finite Hamiltonian $H \in(-\infty, \infty)$ with

$$
H=\frac{1}{2} S_{p}-\frac{g}{\sigma+1} \int \rho^{\sigma+1} d \mathbf{x}, \quad \rho(z, \mathbf{x})=\int W(z, \mathbf{x}, \mathbf{p}) d \mathbf{p}
$$

The first term $S_{p}$ in $H$ is the kinetic energy and the second term is the (self-interaction) potential energy. Note that in the presence of a random potential, the value of the Hamiltonian (11) is not conserved under the evolution (5). Indeed, the ensemble-averaged value of the Hamiltonian increases in time, cf. (29) below. 


\section{ENERGY AND VARIANCE IDENTITY}

Let us analyze the evolution of the mean Hamiltonian $\langle H\rangle$ and the average spread $\left\langle S_{x}\right\rangle$. First note the result of $\mathcal{U}_{\gamma}$ acting on the quadratic polynomials:

$$
\begin{aligned}
\mathcal{U}_{\gamma} \mathbf{x} & =0 \\
\mathcal{U}_{\gamma} \mathbf{p} & =i \int e^{i \mathbf{q} \cdot \mathbf{x}} \mathbf{q} \hat{U}(\mathbf{q}) d \mathbf{q}=\nabla_{\mathbf{x}} U \\
\mathcal{U}_{\gamma}|\mathbf{x}|^{2} & =0 \\
\mathcal{U}_{\gamma} \mathbf{x} \cdot \mathbf{p} & =i \int e^{i \mathbf{q} \cdot \mathbf{x}} \mathbf{x} \cdot \mathbf{q} \hat{U}(\mathbf{q}) d \mathbf{q}=\mathbf{x} \cdot \nabla_{\mathbf{x}} U \\
\mathcal{U}_{\gamma}|\mathbf{p}|^{2} & =i \int e^{i \mathbf{q} \cdot \mathbf{x}} 2 \mathbf{p} \cdot \mathbf{q} \hat{U}(\mathbf{q}) d \mathbf{q}=2 \mathbf{p} \cdot \nabla_{\mathbf{x}} U .
\end{aligned}
$$

A remarkable fact is that these results are independent of $\gamma \geq 0$.

Using (12)-(16) and (6) we obtain by integrating eq. (5) that

$$
\begin{aligned}
\frac{\partial}{\partial z}\left\langle S_{p}\right\rangle & =\left\langle\int \nabla_{\mathbf{x}} U \cdot 2 \mathbf{p} W d \mathbf{x} d \mathbf{p}\right\rangle+\int \mathcal{Q}_{\gamma}|\mathbf{p}|^{2}\langle W\rangle d \mathbf{p} d \mathbf{x} \\
\frac{\partial}{\partial z} \frac{g}{\sigma+1} \int\left\langle\rho^{\sigma+1}\right\rangle d \mathbf{x} & =\left\langle\int \nabla_{\mathbf{x}} U \cdot \mathbf{p} W d \mathbf{p} d \mathbf{x}\right\rangle .
\end{aligned}
$$

from which the evolution equation for the mean value of the Hamiltonian follows

$$
\frac{\partial}{\partial z}\langle H\rangle=\int \mathcal{Q}_{\gamma}|\mathbf{p}|^{2}\langle W\rangle d \mathbf{p} d \mathbf{x}
$$

We shall refer to (17) as the energy law.

4.1. Variance identity. The variance identity has been long used to derive the wave collapse condition for the NLS in the homogeneous case [13. Below we reformulate it for the phase space evolution eq. (5).

We have another set of remarkably simple properties of $\mathcal{Q}_{\gamma}$ independent of $\gamma \geq 0$ :

$$
\begin{aligned}
\mathcal{Q}_{\gamma} \mathbf{x} & =0 \\
\mathcal{Q}_{\gamma} \mathbf{p} & =\int \Phi(\mathbf{p}-\mathbf{q})(\mathbf{p}-\mathbf{q}) d \mathbf{q}=0 \quad(\text { by (22) }) \\
\mathcal{Q}_{\gamma}|\mathbf{x}|^{2} & =0 \\
\mathcal{Q}_{\gamma} \mathbf{x} \cdot \mathbf{p} & =\mathbf{x} \cdot \int \Phi(\mathbf{p}-\mathbf{q})(\mathbf{p}-\mathbf{q}) d \mathbf{q}=0 \quad(\text { by (2) }) \\
\mathcal{Q}_{\gamma}|\mathbf{p}|^{2} & =-\int \Phi(\mathbf{p}-\mathbf{q})\left[|\mathbf{p}|^{2}-|\mathbf{q}|^{2}\right] d \mathbf{q} \\
& =-\int \Phi(\mathbf{q})(2 \mathbf{p}-\mathbf{q}) \cdot \mathbf{q} d \mathbf{q} \\
& =\int \Phi(\mathbf{q})|\mathbf{q}|^{2} d \mathbf{q} \equiv 2 R .
\end{aligned}
$$

For the diffusion operator $\mathcal{Q}_{0}$ we have $R=2 \times \operatorname{tr}(\mathbf{D})$ where the diffusion matrix $\mathbf{D}$ is given by (7). We shall use the above identities to perform integrating by parts in the derivation of the variance identity. 
$\left\langle S_{x}\right\rangle$

Combining the results from the previous section and (18)-(24) we obtain the rate of change of

$$
\frac{\partial}{\partial z}\left\langle S_{x}\right\rangle=2\left\langle S_{x p}\right\rangle
$$

where $S_{x p}$ is the cross-moment

$$
S_{x p}=\int \mathbf{x} \cdot \mathbf{p} W d \mathbf{x} d \mathbf{p}
$$

Differentiating $S_{x p}$ and taking expectation we obtain

$$
\frac{\partial}{\partial z}\left\langle S_{x p}\right\rangle=\left\langle S_{p}\right\rangle-\frac{g d \sigma}{\sigma+1} \int\left\langle\rho^{\sigma+1}\right\rangle d \mathbf{x} d \mathbf{p}
$$

Hence the second derivative of $S_{x}$ becomes

$$
\frac{\partial^{2}}{\partial z^{2}}\left\langle S_{x}\right\rangle=4\langle H\rangle+\frac{2(2-d \sigma) g}{\sigma+1} \int\left\langle\rho^{\sigma+1}\right\rangle d \mathbf{x} .
$$

Alternatively using the definition of $H$ we can rewrite the variance identity (26) as

$$
\frac{\partial^{2}}{\partial z^{2}}\left\langle S_{x}\right\rangle=2 d \sigma\langle H\rangle+(2-d \sigma)\left\langle S_{p}\right\rangle
$$

which in the critical case $d \sigma=2$ becomes

$$
\frac{\partial^{2}}{\partial z^{2}}\left\langle S_{x}\right\rangle=2 d \sigma\langle H\rangle .
$$

Both (26) and (27) will be useful for estimating wave spread.

\section{Dispersion RATE}

Although the medium is lossless, reflected in the fact that the total mass $N=1$ is conserved, the value of the Hamiltonian, however, is not conserved by the evolution since the random scattering is not elastic due to time-varying nature of the random potential. Indeed, by (17) and (24), the average Hamiltonian is an increasing function of time

$$
\frac{\partial}{\partial z}\langle H\rangle=R, \quad\langle H\rangle(z)=H(0)+R z
$$

due to the diffusion-like spread in the momentum $\mathbf{p}$.

In the critical case $d \sigma=2$, we obtain from eq. (28) and (29) the exact result

$$
\frac{\partial^{2}}{\partial z^{2}}\left\langle S_{x}\right\rangle=4\langle H\rangle=4 H(0)+4 R z
$$

before any singularity formation causes a possible breakdown of the variance identity. Integrating (30) twice we obtain the exact spread rate as stated below.

Proposition 1. If $d \sigma=2$ or $g=0$, then

$$
\left\langle S_{x}\right\rangle(z)=S_{x}(0)+2 S_{x p}(0) z+2 H(0) z^{2}+\frac{2 R}{3} z^{3} .
$$


The analogous result $\left(S_{x} \sim z^{3}\right)$ for the linear Schrödinger equation $(d=1, g=0)$ with a random potential has been proved previously [1, 3.

Next we consider the supercritical case and the defocusing case. We have from (26) that

$$
\frac{\partial^{2}}{\partial z^{2}}\left\langle S_{x}\right\rangle=4\langle H\rangle+\frac{(4-2 d \sigma) g}{\sigma+1} \int\left\langle\rho^{\sigma+1}\right\rangle d \mathbf{x}
$$

and hence

$$
\begin{array}{ll}
\frac{\partial^{2}}{\partial z^{2}}\left\langle S_{x}\right\rangle \leq 4\langle H\rangle=4 H(0)+4 R z, \quad \text { for } g(2-d \sigma)<0 \\
\frac{\partial^{2}}{\partial z^{2}}\left\langle S_{x}\right\rangle \geq 4\langle H\rangle=4 H(0)+4 R z, \quad \text { for } g(2-d \sigma) \geq 0 .
\end{array}
$$

On the other hand, from (27) we obtain for any $g$

$$
\begin{aligned}
& \frac{\partial^{2}}{\partial z^{2}}\left\langle S_{x}\right\rangle \leq 2 d \sigma\langle H\rangle, \quad \text { for } 2-d \sigma \leq 0 \\
& \frac{\partial^{2}}{\partial z^{2}}\left\langle S_{x}\right\rangle \geq 2 d \sigma\langle H\rangle, \quad \text { for } 2-d \sigma \geq 0 .
\end{aligned}
$$

Integrating the above inequalities twice, we obtain the following.

Proposition 2. The following estimates hold

$$
\begin{array}{ll}
\left\langle S_{x}(z)\right\rangle \leq S_{x}(0)+2 S_{x p}(0) z+2 H(0) z^{2}+\frac{2}{3} R z^{3}, & g(2-d \sigma) \leq 0 \\
\left\langle S_{x}(z)\right\rangle \geq S_{x}(0)+2 S_{x p}(0) z+2 H(0) z^{2}+\frac{2}{3} R z^{3}, & g(2-d \sigma) \geq 0
\end{array}
$$

and

$$
\begin{aligned}
& \left\langle S_{x}(z)\right\rangle \leq S_{x}(0)+2 S_{x p}(0) z+d \sigma H(0) z^{2}+\frac{d \sigma}{3} R z^{3}, \quad 2 \leq d \sigma \\
& \left\langle S_{x}(z)\right\rangle \geq S_{x}(0)+2 S_{x p}(0) z+d \sigma H(0) z^{2}+\frac{d \sigma}{3} R z^{3}, \quad 2 \geq d \sigma .
\end{aligned}
$$

Therefore

Corollary 1. Assume $g<0$ (hence $H \geq 0$ ). Then

$$
\left\langle S_{x}(z)\right\rangle \leq S_{x}(0)+2 S_{x p}(0) z+(d \sigma \vee 2) H(0) z^{2}+\frac{d \sigma \vee 2}{3} R z^{3}
$$

and

$$
\left\langle S_{x}(z)\right\rangle \geq S_{x}(0)+2 S_{x p}(0) z+(d \sigma \wedge 2) H(0) z^{2}+\frac{d \sigma \wedge 2}{3} R z^{3} .
$$

In other words the variance $\left\langle S_{x}\right\rangle$ is cubic-in-time in the defocusing case. In the subcritical case the cubic law is a lower bound while in the supercritical case the cubic law is an upper bound. 


\section{Finite-time Singularity}

Finite-time singularity for the critical or supercritical $(d \sigma \geq 2)$ self-focusing NLS equation in the homogeneous case is a well known effect [13. In this case the singularity is the blow-up type $S_{p}, \int|\rho|^{\sigma+1} \rightarrow \infty$. Here we call breakdown of the variance identity (26)-(27) or the energy law (17) as finite-time singularity and seek the sufficient conditions for singularity with positive probability. We show that in the supercritical case with additional assumptions the finite-time singularity is of the blow-up type in the sense that $\left\langle S_{p}\right\rangle, \int\left\langle\rho^{\sigma+1}\right\rangle$ tend to infinity. As such the blow-up phenomenon discussed here is not necessarily a sure event but rather an event of a positive probability that $S_{p}$ and $\int \rho^{\sigma+1}$ can exceed any fixed level in a finite time.

For $g \geq 0, d \sigma \geq 2$ one can bound $S_{x}$ as in the inequality (35)

$$
\left\langle S_{x}(z)\right\rangle \leq S_{x}(0)+2 S_{x p}(0) z+d \sigma H(0) z^{2}+\frac{d \sigma R}{3} z^{3} \equiv F(z)
$$

and as motivated by the homogeneous case we look for the conditions when $F(z)$ vanishes at a finite $z \geq 0$.

A sufficient condition for $F(z)$ to vanish at a finite positive $z$ can be derived from that $F(z)$ takes a non-positive value $F\left(z_{0}\right) \leq 0$ at its local minimum point $z_{0}>0$. The local minimum point $z_{0}$ is given by

$$
z_{0}=\frac{-H(0)+\sqrt{H(0)^{2}-2 R S_{x p}(0) /(d \sigma)}}{R} .
$$

Therefore we are led to the following conditions for singularity.

Proposition 3. Assume $d \sigma \geq 2, g>0$. The solutions of the NWMI or NLI equation develop singularities with positive probability at a finite time $z_{*} \leq z_{0}$ given by (39) under the condition $F\left(z_{0}\right) \leq 0$ and either one of the following conditions

$$
\begin{aligned}
& S_{x p}(0)<0 \\
& S_{x p}(0)>0, \quad H(0)<-\sqrt{\frac{2 R S_{x p}(0)}{d \sigma}} .
\end{aligned}
$$

Remark 1. Clearly, the condition $F\left(z_{0}\right) \leq 0$ requires $H(0)$ to be sufficiently below $\bar{H}$ by allowing the self-interaction energy

$$
-\frac{g}{\sigma+1} \int \rho^{\sigma+1}(0) d \mathbf{x} d \mathbf{p}
$$

to be sufficiently negative.

Remark 2. Using, instead, inequality (33) one can obtain an alternative expression

$$
z_{0}=\frac{-H(0)+\sqrt{H(0)^{2}-R S_{x p}(0)}}{R}
$$

and the corresponding conditions for singularity formation, namely either $S_{x p}(0)<0$ or $S_{x p}(0)>$ $0, H(0)<-\sqrt{R S_{x p}(0)}$.

It may be the case that for a given initial condition the solutions develop singularity at different times depending on the realization of random potential. To analyze such an effect we need the probabilistic versions of variance identity and energy law which are much more involved. 
6.1. Blow-up. We will follow the argument of [7] to show more explicitly the blow-up mechanism in the case with the supercritical, self-focusing nonlinearity and give a sharper bound on $z_{*}$ under certain circumstances.

Proposition 4. Suppose $d \sigma>2, g>0$. Then under the conditions (40) and

$$
\left\langle H\left(z_{*}\right)\right\rangle=H(0)+R z_{*} \leq 0, \quad \text { with } z_{*}=\frac{2 S_{x}(0)}{S_{x p}(0)(2-d \sigma)},
$$

$\partial\left\langle S_{x}\right\rangle / \partial z$ and $\left\langle S_{p}\right\rangle$ blow up before or at a finite time $z_{*}$.

Proof. Since blow-up is a local phenomenon, $S_{x}$ is a poor indicator of its occurrence. A more relevant object to consider is $S_{p}$. From (27) it follows that before $z_{*}$

$$
\frac{\partial^{2}}{\partial z^{2}}\left\langle S_{x}\right\rangle=2 \frac{\partial}{\partial z}\left\langle S_{x p}\right\rangle \leq(2-d \sigma)\left\langle S_{p}\right\rangle<0 .
$$

Since $\partial\left\langle S_{x}\right\rangle / \partial z=2\left\langle S_{x p}\right\rangle<0$ by (44) and (40), $\partial\left\langle S_{x}\right\rangle / \partial z$ is a negative, decreasing function up to the time $z_{*}$. Hence we have

$$
0 \leq\left\langle S_{x p}\right\rangle^{2} \leq\left\langle S_{x}\right\rangle\left\langle S_{p}\right\rangle \leq S_{x}(0)\left\langle S_{p}\right\rangle, \quad z \in\left[0, z_{*}\right]
$$

which implies

$$
\left\langle S_{p}\right\rangle \geq \frac{\left\langle S_{x p}\right\rangle^{2}}{S_{x}(0)}
$$

Let $A(z)=-\left\langle\partial S_{x}(z) / \partial z\right\rangle \geq 0, z \leq z_{*}$. We have from (44), (46) and (25) the differential inequality

$$
\frac{\partial}{\partial z} A \geq C A^{2}, \quad C=\frac{d \sigma-2}{4 S_{x}(0)}>0
$$

which yields the estimate

$$
A(z) \geq \frac{A(0)}{1-z C A(0)}, \quad z<\frac{1}{C A(0)}=z_{*}
$$

and thus the blow-up of $A(z)$ before or at $z_{*}$. This along with (46) then implies the blow-up of $S_{p}$ at a finite time.

The preceding argument demonstrates clearly the blow-up mechanism, namely the quadratic growth property (47).

Remark 3. It should be noted that, since $H\left(z_{0}\right)>0$, the condition 43 implies that $z_{*}<z_{0}$, as given by either (39) or (42). Therefore, under the condition (43), $z_{*}$ provides a sharper upper bound on the time of singularity than $z_{0}$.

Remark 4. Since $\langle H(z)\rangle$ is bounded over compact sets of $z$, Proposition 4 implies the blow-up of the self-interaction energy $\int\left\langle\rho^{\sigma+1}\right\rangle$ in a finite time. 


\section{Conclusion}

We have presented an elementary approach for analyzing the nonlinear Schrödinger equation with a white-noise potential. We have focused on the ensemble-averaged quantities such as the variance identity and energy law in order to shed light on two problems: the rate of spread and the singularity formation.

We have shown that the ensemble-averaged spread in the critical or defocusing case follows the cubic-in-time law while in the supercritical and subcritical focusing cases the cubic law becomes an upper and lower bounds respectively. In a separate publication we will use these estimates to analyze the limitation on channel capacity in optical fibers due to self-phase and cross-phase modulations 8 .

We have also found singularity conditions in the critical and supercritical focusing cases. And we show the finite-time singularity in the supercritical case is of the blow-up type. The singularity/blowup discussed in the present paper is not necessarily a sure event but that of a positive probability.

\section{REFERENCES}

[1] L. Bunimovich, H.R. Jauslin, J.L. Lebowitz, A. Pellegrinotti and P. Nielaba, Diffusive energy growth in classical and quantum driven oscillators. J. Stat. Phys.62(1991), 793-817.

[2] A. Debussche and L. Di Menza, Numerical simulation of focusing stochastic nonlinear Schrödinger equations. Phys. D 162 (2002), 131-154.

[3] E.B. Erdogan, R. Killip and W. Schlag, Energy growth in Schrödinger's equation with Markovian driving. Commun. Math. Phys.240(2003), 1-29.

[4] A. Fannjiang, White-noise and geometrical optics limits of Wigner-Moyal equation for wave beams in turbulent media. Comm. Math. Phys.254:2 (2005), 289-322.

[5] A. Fannjiang, Self-averaging in scaling limits for random parabolic waves. Arch. Rat. Mech. Anal.175:3 (2005), $343-387$.

[6] P. Gérard, P.A. Markowich, N.J. Mauser and F. Poupaud, Homogenization limits and Wigner transforms, Comm. Pure Appl. Math. I (1997), 323-379.

[7] R.T. Glassey, On the blow-up of solutions to the Cauchy problem for nonlinear Schrödinger equations, J. Math. Phys. 18(1977), 1794-1797.

[8] L. Kazovsky, S. Benedetto, A. Willner, Optical Fiber Communication Systems, Artech House, 1996.

[9] P.L. Lions and T. Paul, Sur les mesures de Wigner. Rev. Mat. Iberoamericana 9(1993), 553-618.

[10] P.P. Mitra and J.B. Stark, Nonlinear limits to the information capacity of optical fibre communications, Nature 411 (2001), 1027-1030.

[11] A. C. Newell and J. V. Moloney, Nonlinear Optics, Addison-Wesley, Redwood City, CA., 1992.

[12] G. Papanicolaou and W. Kohler, Comm. Pure Appl. Math. 27 (1974), 641-668.

[13] C. Sulem and P.-L. Sulem, The Nonlinear Schrödinger Equation: Self-Focusing and Wave Collapse. Springer, New York, 1999.

[14] S. Tcheremchantsev, Transport properties of Markovian Anderson model, Commun. Math. Phys.196:1 (1998), 105-131.

E-mail address: cafannjiang@ucdavis.edu

Department of Mathematics, University of California, Davis 95616-8633 